\title{
KLAUS-DIETER LUDWIG
}

\section{Stilkennzeichnungen und Stilbewertungen in deutschen Wörterbüchern der Gegenwart.}

\section{Vorbemerkung}

Es wird berichtet, daß die Mitherausgeberin des "Wörterbuch[es] der deutschen Gegenwartssprache", Ruth Klappenbach, bei einem Besuch in der ČSSR freundlich mit den Worten begrüßt wurde: „Ich glaube, daß ich Ihre Visage schon einmal gesehen habe." Müller (1984) verzeichnet diese Begebenheit im Zusammenhang mit Fehlleistungen in der Wortwahl und bemerkt: „Die korrekte Anwendung der Wörter bereitet sowohl Ausländern als auch Muttersprachlern nicht selten Schwierigkeiten" (S. 366). Korrekte Anwendung wird hier in Verbindung gebracht mit einem "Zusatz”, der zu einer im Wörterbuch kodifizierten lexikalischen Einheit steht oder nicht steht.

Schlägt man in allgemeinen einsprachigen Bedeutungswörterbüchern der deutschen Gegenwartssprache nach, so konstatiert man in sieben Wörterbüchern dieses Typs drei unterschiedliche „Zusätze” beim Lemma Visage ('Gesicht'):

"umgangssprachlich" (Wahrig-DW, Knaur),

"salopp" (WDG, Duden-GWB, Duden-UW, HDG),

"derb" (Duden-Bedeutungswörterbuch).

Nimmt man noch die drei letzten Auflagen des Rechtschreib-Dudens hinzu, so begegnet man hier zwei verschiedenen "Zusätzen" bei Visage: "umgangssprachlich" (Duden Mannheim 1986; Duden Mannheim/Leipzig 1991), „salopp” (Duden Leipzig 1985).

Übereinstimmend in allen genannten Wörterbüchern - den sieben allgemeinen einsprachigen und den drei Dudenausgaben - ist der weitere Zusatz „abwertend” (im Duden Mannheim 1986 „verächtlich"; im HDG "/emot. neg./"). Es sei darauf aufmerksam gemacht, $\mathrm{daB}$ fünf der genannten Wörterbücher (Duden-GWB, Duden-UW, Duden-Bedeutungswörterbuch, Duden Mannheim 1986, Duden Mannheim/Leipzig 1991) unter der Verantwortung einer Redaktion (an einem Ort) entstanden sind und daß sich in drei dieser fünf Wörterbücher ganz unterschiedliche „Zusätze” bei einem Lemma finden.

Mit diesem Beispiel wollte ich zeigen, worauf das Augenmerk der folgenden Darlegungen gerichtet werden soll: auf "Zusätze” der erwähnten Art, auch „Markierungen”, „Markierungsprädikate” oder "Marker” genannt; Hausmann spricht von "Markierungsetiketten” (1989, S. 649); auf die 
sogenannte "stilistische Charakterisierung des deutschen Wortschatzes" (WDG, S. 011) im Wörterbuch, auf Stilkennzeichnungen und Stilbewertungen - oder besser gesagt - Stilkennzeichnungen durch Stilbewertung.

Während die meisten Beiträge zu „Stilfragen” den Gegenstand „Stil” insbesondere unter theoretischem Aspekt erörtert haben, möchte ich mich ausschlieBlich auf "Stilfragen" der lexikographischen Praxis konzentrieren. Zunächst gilt die Aufmerksamkeit den Markierungskategorien 'Stilschicht'/'Stilebene' und 'Stilfärbung' im allgemeinen (1.). Danach sollen Unterschiede und Veränderungen der stilistischen Charakterisierung von lexikalischen Einheiten in verschiedenen deutschen Gegenwartswörterbüchern dargestellt werden (2.). Schließlich wollen wir thesenhaft einen Ausblick auf eine mögliche Verbesserung sogenannter stilistischer Markierungen in der lexikographischen Praxis geben (3.).

\section{Zu den Markierungskategorien „Stilschicht" / Stilebene” und „Stilfärbung"}

\subsection{Ein Blick zurück}

DaB die lexikographische Methode, in einem Wörterbuch verzeichnete lexikalische Einheiten „relativ zu z w e i Bezugssystemen bewertend zu kennzeichnen" (Wiegand 1981, S. 148), in der germanistischen Lexikographie eine lange Tradition hat, ist weithin bekannt. Dies soll hier nicht im einzelnen wiederholt und dargelegt werden (s. dazu Wiegand 1981; Ludwig 1991). Aber es sei vermerkt, daß nicht erst mit dem „Wörterbuch der deutschen Gegenwartssprache" die systematische „stilistische Bewertung" des in einem einsprachigen synchronischen Wörterbuch erfaßten Wortschatzes begonnen hat. Aussagen, daß man vor Erscheinen des WDG stilistische Markierungen "nur unsystematisch und sporadisch angebracht” habe (Püschel 1989, S. 694; vgl. auch Klappenbach †/MaligeKlappenbach 1978, S. 27), sind $z u$ relativieren. Es waren vor allem Adelung und Campe, die sich nicht mit sporadischen und unsystematischen Hinweisen als lexikographische Angaben zum Stil begnügten. Adelung benutzt ein fünfschichtiges System von Schreib- und Sprecharten, nach dem er die "Würde der Wörter" kennzeichnet (Adelung I, 1774, S. XIV). Es sind die "fünf Classen": "höhere oder erhabene Schreibart", "edle” Schreibart, „Sprechart des gemeinen Lebens und vertraulichen Umganges", "niedrige" Sprechart und schließlich die „ganz pöbelhafte” Sprechart (ebd.) - „Classen”, die mit „Stilschichten” bzw. „Stilebenen” der gegenwärtigen deutschen Lexikographie durchaus vergleichbar sind. "Ich habe mich bemühet, in den nöthigsten Fällen (denn in allen konnte es nicht geschehen) die Classe, in welche jedes Wort, oder jede Redensart gehöret, anzugeben" (ebd.), schreibt Adelung in der Vorrede zur 1. Auf- 
lage seines Wörterbuches, das unter dem Titel „Versuch eines vollständigen grammatisch-kritischen Wörterbuches Der Hochdeutschen Mundart, mit beständiger Vergleichung der übrigen Mundarten, besonders aber der Oberdeutschen" 1774-1786 erschienen ist. Sollte damit etwa eine "Classe" angesprochen sein, zu der Wörter gehören, die gewissermaßen als "neutral” oder "normalsprachlich" gelten - wie die heutigen Lexikographen diese "Stilschicht” nennen? In „allen [Fällen] konnte es nicht geschehen die Classe, in welche jedes Wort, oder jede Redensart gehöret, anzugeben" (s.o.). Adelungs Wörterbuch verzeichnet auch Lexeme, die nicht den "fünf Classen" zugeordnet werden.

Was die Charakterisierung einer „Nullmarkierung” betrifft, so ist Campe im Vergleich zu Adelung genauer. Hatte Adelung von der "Würde der Wörter" gesprochen, so nennt dies Campe "die innern Grade ihres Adels oder ihrer Gemeinheit" (Campe I, 1807, S. XIII), und er ist bemüht, „die Würdigung der Wörter” u.a. auch nach „ihrer Brauchbarkeit für die höhere oder niedrigere [...] Schreibart" zu kennzeichnen (ebd.). Er unterscheidet zunächst "Wörter der höhern Schreibart” und "niedrige Wörter”, die er weiter differenziert in: „Niedrige, aber deswegen noch nicht verwerfliche Wörter, weil sie in der geringern (scherzenden, spottenden, launigen) Schreibart, und in der Umgangssprache brauchbar sind [...]"; „Niedrige Wörter, die ans Pöbelhafte grenzen, und deren man sich daher, sowol in der Schriftsprache, selbst in der untern, als auch in der bessern Umgangssprache, enthalten sollte [...]" (Campe I, 1807, S. XXI). Campe verwendet zur stilistischen Charakterisierung und zur Kennzeichnung landschaftlicher, veralteter und neugebildeter Wörter insgesamt 14 „Kürzungszeichen”, die er in der Vorrede zum ersten Band seines "Wörterbuch[es] der Deutschen Sprache" (1807-1811) einführt und ausführlich erläutert (Campe I, 1807, S. XX f.). Wichtig ist der sich hieran anschließende Zusatz: „Wörter, die allgemein üblich sind, und für jede Schreibart passen, haben gar kein Zeichen" (S. XXI). Einige Beispiele für die Bewertungspraxis in Adelungs und Campes Wörterbuch finden sich in Tabelle 1.

Das zweite Bezugssystem sogenannter stilistischer Bewertung von sprachlichen Ausdrücken im Wörterbuch betrifft Markierungsprädikate, die heute zu den „Stilfärbungen” (WDG) oder "Gebrauchsangaben” (Duden-GWB) gezählt werden. Wir finden sie auch bei Adelung und Campe, z.B.:

Mos [...] ein nur in den niedrigen Sprecharten im $\mathrm{S} \mathrm{ch}$ e $\mathrm{r} \mathrm{z}$ e zuweilen übliches Wort [...] und Geld bedeutet. [...] (Adelung III, 1777, Sp. 587) 


\begin{tabular}{|l|c|c|}
\hline $\begin{array}{l}\text { Lemma- } \\
\text { beispiele }\end{array}$ & \multicolumn{2}{|c|}{ Stilistische Markierung in den Wörterbüchern von } \\
\hline & Adelung & Campe \\
\hline dreckig & in den niedrigen Sprecharten & $X$ \\
\hline duzen & $\begin{array}{c}\text { im gemeinen Leben und in } \\
\text { der niedrigen Sprechart }\end{array}$ & $X$ \\
\hline Fenster & - & - \\
\hline Flügel & - & - \\
\hline Fittich & in der höheren Schreibart & $\Delta$ \\
\hline Schwinge & in der dichterischen Schreibart & $\Delta$ in der höhern Schreibart \\
\hline Gefilde & $\begin{array}{c}\text { in der edlen und dichterischen } \\
\text { Schreibart }\end{array}$ & $\triangle$ \\
\hline Hain & $\begin{array}{c}\text { in der höhern und } \\
\text { dichterischen Schreibart }\end{array}$ & $\begin{array}{c}\text { in der höhern dichterischen } \\
\text { Schreibart }\end{array}$ \\
\hline Scheiße & in den niedrigsten Sprecharten & Xpöbelhaftes Wort \\
\hline
\end{tabular}

Tabelle 1: Beispiele für stilistische Markierungen in Adelungs und Campes Wörterbuch

$$
\begin{aligned}
- & =\text { ohne Markierungsprädikat („Nullmarkierung”) } \\
\triangle & =\text { Wörter der höhern Schreibart } \\
X & =\text { Wörter des gemeinen Lebens } \\
\not{X} & =\text { Pöbelhafte Wörter }
\end{aligned}
$$

X - Der Ehekrüppel [...] im gemeinen $\mathrm{S}$ c h e r z, ein alter gebrechlicher, unfähiger Ehemann; dann überhaupt, ein jeder Ehemann. (Campe I, 1807, S. 818)

Dichterling [...] ein schlechter, niedriger Dichter, im ver ä ch tl i c h e n Verstande. [...] Adelung I, 1774, Sp. 1343)

Der Wanst [...] der Schmerbauch an den Thieren, wie auch, aber in ver $\ddot{a} \mathrm{cht} \mathrm{li} \mathrm{ch} \mathrm{e} \mathrm{m} \mathrm{Verstande,} \mathrm{an} \mathrm{dem} \mathrm{Menschen.} \mathrm{[...]} \mathrm{(Campe} \mathrm{V,}$ 1811, S. 571). 


\subsection{Zur Gegenwart}

Die systematische "stilistische Charakterisierung des deutschen Wortschatzes" (WDG, S. 011) in einem einsprachigen synchronischen Wörterbuch ist nach Campe mit dem WDG wieder aufgenommen worden. Mit dem Erscheinen des „Wörterbuch[es] der deutschen Gegenwartssprache" (1961-1977) hat in der germanistischen Lexikographie die Ära der stilistischen Bewertung von Wörtern und ihren Verwendungsweisen durch die Zuordnung zu sogenannten "Stilschichten” (später auch „Stilebenen” genannt, vgl. HDG) und/oder "Stilfärbungen” (entsprechen den "Gebrauchsangaben" in Duden-GWB und Duden-UW) begonnen. Klappenbach schreibt 1964 über das entstehende Werk: „Es ist ein synchronisches Bedeutungswörterbuch, in dem das Einzelwort nach seinem stilistischen Wert bestimmt, in seinen typischen Fügungen vorgeführt und grammatisch gekennzeichnet wird" (zit. nach Klappenbach 1980, S. 90). Sie hebt hervor: „Das Neue und Besondere an diesem Wörterbuch ist die Einordnung des Wortschatzes in ein stilistisch aufgegliedertes Gefüge" (ebd.). Dieses "Gefüge” besteht zunächst aus vier „Stilschichten”. „Zu den Stilschichten treten Stilfärbungen oder Nuancen, die ein Wort oder eine Redewendung erhalten kann", lesen wir weiter bei Klappenbach (ebd., S. 92) - im WDG sind es elf. SchlieBlich: „Stilschicht und Stilfärbung sind e i n e Ebene, auf der wir den Wortschatz betrachten. Diese Stilanalyse [...] ist noch in keinem bisherigen deutschen Wörterbuch konsequent durchgeführt worden" (ebd.).

Das „stilistisch aufgegliederte Gefüge” des WDG ist Vorbild für andere Wörterbücher der deutschen Gegenwartssprache geworden. Auf die in den verschiedenen Wörterbüchern benutzten Markierungsprädikate für „Stilschichten" / Stilebenen" und „Stilfärbungen" und die dazu gegebenen Erläuterungen - wenn überhaupt vorhanden - soll hier nicht im einzelnen eingegangen werden. Wir haben uns an anderer Stelle ausführlicher zum Status und zur Praxis dieser Markierungen geäußert (vgl. Ludwig 1991).

Als „stilistische Bewertungen von Wörtern und Verwendungsweisen” werden zunächst Markierungsprädikate oder Marker verwendet, die zu „Stilschichten” bzw. „Stilebenen” gezählt werden, metalexikographisch diastratische Markierungen genannt. Ausgangspunkt für die Zuordnung zur Stilschicht sind „die normalsprachlichen Wörter [...], die in ihrem Stilwert neutral sind" (Duden-GWB 1976, S. 15; Duden-GWB 1993, S. 19) und die im Wörterbuch nicht besonders gekennzeichnet werden, also „Nullmarkierung” aufweisen. Die „normalsprachliche Schicht [...] wird bei gefühlsmäßig neutraler Haltung verwendet, erscheint im schriftlichen und mündlichen Gebrauch und ist im öffentlichen Leben (im wei- 
testen Sinne) allgemein üblich" (WDG, S. 012). Im übrigen habe ich bisher in keinem Wörterbuch normalsprachlich als Lemma gefunden. Lexeme, die nicht der "normalsprachlichen Schicht" zugeordnet sind, werden dann mit der entsprechenden Markierung entweder oberhalb dieser Schicht als "bildungssprachlich", „gehoben” oder "dichterisch" gekennzeichnet oder unterhalb der neutralen Schicht als "umgangssprachlich", „salopp", „derb” oder „vulgär” (s. Tabelle 2).

\begin{tabular}{|l|l|l|}
\hline WDG (1961) & $\begin{array}{l}\text { Duden-GWB (1976/1993)/ } \\
\text { Duden-UW (1983/1989) }\end{array}$ & HDG (1984) \\
\hline $\begin{array}{l}\text { Stilschichten } \\
\text { gehoben } \\
\text { (einschl. dichterisch) }\end{array}$ & $\begin{array}{l}\text { Stilschichten } \\
\text { gehobene Sprache } \\
\text { (einschl. dichterisch) } \\
\text { bildungssprachlich } \\
\text { normalsprachlich } \\
\text { normalsprachlich } \\
\begin{array}{l}\text { einschl. umgangs- } \\
\text { sprachlich) } \\
\text { salopp- } \\
\text { umgangsspraclulich } \\
\text { vulgär }\end{array}\end{array}$ & $\begin{array}{l}\text { umgangssprachlich } \\
\text { salopp } \\
\text { derb } \\
\text { vulgär }\end{array}$ \\
\hline
\end{tabular}

Tabelle 2: Markierungen für Stilschichten/Stilebenen in einigen deutschen Wörterbüchern

„Zu diesen stilistischen Bewertungen wie zu den normalsprachlichen Wörtern und Verwendungsweisen können Gebrauchsangaben wie 'scherzhaft, spöttisch, ironisch, abwertend, nachdrücklich, gespreizt, verhüllend, Schimpfwort' u.a. treten", heißt es im "Großen Wörterbuch der deutschen Sprache in sechs Bänden" (1976, S. 15). In der neuen achtbändigen Ausgabe folgt der Zusatz: "die [Gebrauchsangaben] etwas über die Haltung des Sprechers oder die Nuancierung einer Äußerung aussagen" (1993, S. 20) - „Gebrauchsangaben”, die im „Wörterbuch der deutschen Gegenwartssprache" als "Stilfärbungen" in Erscheinung treten, metalexikographisch als (dia)evaluative Markierungen bezeichnet.

Was ist eigentlich mit "Stilschicht" bzw. "Stilebene" und mit „Stilfärbung" gemeint? Anzunehmen wäre, daß beide Markierungskategorien in den Wörterbucheinleitungen bzw. Benutzungshinweisen thematisiert werden, man sie zumindest in den jeweiligen Wörterverzeichnissen als Lemmata mit einer Bedeutungserläuterung finden sollte. Weit gefehlt! Doch kehren wir noch einmal zu der von Klappenbach erwähnten „Stilanalyse" zurück. „Stilschicht und Stilfärbung sind e i n e Ebene”, 
auf der der Wortschatz im WDG betrachtet wird, und diese Betrachtung wird als „Stilanalyse” charakterisiert.

Ohne mich mit dem Terminus "Stil” und mit „Stilanalyse” in diesem Zusammenhang zu befassen, sei dazu nur soviel angemerkt: Klappenbach bezieht "Stilanalyse" nicht auf Texte, sondern speziell auf die Zuordnung der im Wörterbuch verzeichneten Wörter zu Stilschichten und Stilfärbungen. Stilanalyse erscheint als Lemma im WDG mit der Bedeutungserläuterung "Analyse einer sprachlichen (schriftlichen) Ausdrucksform" und den lexikographischen Beispielen: eine $S$. anfertigen; in der S. wurden Verstöße gegen die Syntax aufgezeigt. Im Duden-GWB ist Stilanalyse ohne Erklärung verzeichnet. Bleibt noch die sich hier wohl folgerichtig anschließende Frage: Welche Bedeutungserläuterung finden wir unter dem Lemma Stil, die hier in Frage kommt? Ich bleibe also ausschließlich beim Wörterbuch. Im WDG: „1. für eine bestimmte Epoche, Menschengruppe, Einzelperson, einen Lebens-, Tätigkeitsbereich typische, charakteristische (künstlerische) Ausdrucksform, Erscheinungsweise”, wobei dann differenziert wird „a) /mit Bezug auf Sprache, Literatur/" und „b) /mit Bezug auf bildende Kunst, Musik/". Die unter a) zu Stil angegebenen lexikographischen Beispiele (ein guter, gewandter, gepflegter, manierierter, schwülstiger, geschraubter S.; der Schriftsteller schreibt, hat einen eigenwilligen, lebendigen, glänzenden $S$. u.a.) und ebenso die zu Stilanalyse verzeichneten Beispiele lassen darauf schlieBen, daB Stil bzw. Stilanalyse auf einen Text in seiner Gesamtheit und nicht auf ein Einzelwort bezogen wird.

Aber was bedeuten "Stilschicht” / Stilebene" und „Stilfärbung”? Befragt man auch hierzu die Wörterbücher, die beide Bewertungskategorien benutzen und ihnen Markierungen subsumieren, so ist die Ausbeute der Antworten gering.

Das "Wörterbuch der deutschen Gegenwartssprache”, das die Termini "Stilschicht" und "Stilfärbung" in die lexikographische Praxis des Deutschen eingeführt hat, nach denen der kodifizierte Wortschatz mit deskriptiver Absicht stilistisch bewertet wird, erklärt beide Termini weder in der Wörterbucheinleitung, noch erscheinen sie als Lemmata im Wörterverzeichnis. Duden-GWB (1976/1993) und Duden-UW (1983/1989), die ebenfalls den Wortschatz „Stilschichten” zuordnen, erklären den Terminus "Stilschicht" in den Benutzungshinweisen nicht, erfassen ihn aber im Wörterverzeichnis, und zwar auf folgende Weise: Stilschicht hat die Fachgebietszuweisung "Sprachwissenschaft" und wird auf Stilebene verwiesen („vgl. -ebene"), und bei Stilebene findet man die Zuweisung „bes. Sprachwissenschaft” und die Erklärung „bestimmte Ebene des Stils". Die entsprechende Bedeutungserläuterung zu Stil lau- 
tet in beiden Wörterbüchern: "[durch Besonderheiten geprägte] Art u. Weise, etw. mündlich od. schriftlich auszudrücken, zu formulieren". Fündig in bezug auf eine Erklärung beider Bewertungskategorien wird man eigentlich, was die allgemeinen einsprachigen Wörterbücher des Deutschen angeht, nur im „Handwörterbuch der deutschen Gegenwartssprache” (1984): „Die Stilebene wird als eine innerhalb eines Kommunikationsbereichs wegen ihrer Eignung bevorzugte Möglichkeit der Sprachverwendung definiert. Das Wörterbuch gibt die als dominierend eingeschätzte Stilebene an” (S. XXII); „Die Stilfärbung wird verstanden als eine innerhalb eines Kommunikationsbereichs in Verbindung mit der Stilebene wegen ihrer Eignung bevorzugte Möglichkeit spezieller Sprachverwendung. Stilfärbungen drücken spezielle Nuancen aus, mit ihnen werden zusätzlich Gebrauchshinweise gegeben" (S. XXIII).

Wir finden diese Termini zum Beispiel auch in einigen Nachschlagewerken zur Sprachwissenschaft mit folgenden Erklärungen:

Für Krahl/Kurz (1984) ist im „Kleinen Wörterbuch der Stilkunde” Stilschicht - synonym mit Stilsphäre gebraucht - die „Höhenlage sprachlicher Formen im Verhältnis zur literarischen Norm; ästhetische Qualität. Eine mögliche Einteilung ist z.B. die Schichtung vulgär - umgangssprachlich - einfach-literarisch - gehoben - poetisch" (S. 121). Davon unterschieden wird die Stilebene als „durchschnittliches sprachästhetisches $\mathrm{Ni}$ veau eines Aussagekomplexes, eines Textes. Die Stilebene wird von der allgemeinen Stilschicht der Wörter und ihrer Fügung und durch den gesamten Charakter des Textes, z.B. durch dessen intellektuelle Qualität, bestimmt" (S. 113). Stilfärbung, gleichgesetzt mit Stilkolorit, bedeutet „übliche, d.h. im Wörterbuch bereits fixierte stilistische Nuance von Wörtern und Fügungen" (ebd.). Dabei können nach Krahl/Kurz (ebd., S. 113f.) Wörter und Fügungen z.B. aufweisen: ein "spezielles Kolorit" (sie können abwertend, grob, scherzhaft, spöttisch, übertreibend, vertraulich, verhüllend, zotig sein); ein "landschaftliches (territoriales) Kolorit" (z.B. Wiese/Alm in der Lexik; ist/hat gestanden in der Syntax); ein „politisches und soziales Kolorit” (z.B. Establishment/führende Klasse).

In dem von Conrad (1985) herausgegebenen „Lexikon sprachwissenschaftlicher Termini" werden Stilschicht, Stilebene und Stilsphäre als Synonyme behandelt und erklärt als „allgemeine emotionale Höhenlage sprachlicher Mittel; ausgehend von einer neutralen Grundschicht (normalsprachlicher Schicht mit expressiver Nullfärbung), sind nach oben und unten weitere Stilschichten zu unterscheiden" (S. 232). Bei Stilfärbung steht: „konnotative Qualitäten der Rede, die von sachlich neutraler Aussage abweichen und der sprachlichen Äußerung 
zusätzlich einen bestimmten Grad von Expressivität verleihen" (ebd., S. 231). Hier ist neben den bekannten Stilfärbungen "vertraulich" (Alterchen), "gespreizt" (Beinkleid), "abwertend" (klauen), „spöttisch" (neunmalklug), „übertrieben” (sich vor Ärger die Haare raufen), „verhüllend" (abberufen werden für sterben) auch von nzeitlicher" (veraltet: Eidam, veraltend: Gendarm u.a.), "territorialer" (Schlächter, Metzger; Schrippe) und "sozialer" Stilfärbung (Jargonismen: kesse Biene, Moos für Geld) die Rede.

Im „Sachwörterbuch für die deutsche Sprache” (1989) wiederum werden Stilfärbung und Stilschicht synonym behandelt und aufgefaßt als "die Summe von (sachbedingt oder einstellungsbedingt) wertenden und von situativen Konnotationen sprachlicher Mittel" (S. 240). In neueren linguistischen Nachschlagewerken (z.B. BuBmann 1990, Lewandowski 1990, Metzler-Lexikon Sprache 1993) tauchen Stilschicht/ Stilebene und Stilfärbung als Lemmata nicht auf.

Ich möchte die unterschiedlichen Auffassungen nicht weiter kommentieren, es sollte damit lediglich auf das terminologische Dilemma in bezug auf "Stilschicht" / Stilebene" und "Stilfärbung” im allgemeinen hingewiesen werden. Es gibt keine allgemein verbindliche Definition dieser Markierungskategorien, geschweige denn der ihnen zugeordneten Markierungsprädikate. Hinzu kommt, daß einerseits die Zahl der Markierungen von Wörterbuch zu Wörterbuch schwankt und andererseits die Zuordnung einzelner Markierungen entweder zu „Stilschichten” oder zu "Stilfärbungen" differiert.

\section{Unterschiede und Veränderungen der stilistischen Bewertung von lexikalischen Einheiten in deutschen Gegenwartswörterbüchern}

\subsection{Zur Markierungspraxis in allgemeinen einsprachigen Wörterbüchern}

Es ist auffällig, daß das Markierungsprädikat „derb” im WDG als Stilfärbung, in Duden-GWB und Duden-UW als Stilschicht in Erscheinung tritt. Die Anwendung von "derb" wird im WDG nicht näher erläutert. In Duden-GWB und Duden-UW gehört „derb” zur Schicht unterhalb der normalsprachlichen Schicht und gilt für "Wörter, die einer groben und gewöhnlichen Ausdrucksweise angehören", wie z.B. Arsch, bescheißen, Fresse (Duden-GWB 1993, S. 20; vgl. auch DudenUW 1989, S. 9). Während "umgangssprachlich”, "salopp”, „derb” und "vulgär" in den beiden zuletzt genannten Wörterbüchern gewissermaßen als „Graduierungen” zu einer Schicht unterhalb der normalsprachlichen 
gehören - „reicher gegliedert ist die Schicht unterhalb der normalsprachlichen Stilschicht" (Duden-GWB 1993, S. 20) -, begreift das WDG "urmgangssprachlich" als "Variante der Normalsprache" und "salopp" (hier: „salopp-umgangssprachlich”) und „vulgär” als eigenständige Stilschichten (WDG, S. 012). Die im WDG als "vulgär" gekennzeichnete unterste Ebene erscheint im HDG als "derb”. In Duden-GWB (1976, S. 15/1993, S. 19) und Duden-UW (1989, S. 9) wird oberhalb der normalsprachlichen Schicht "eine gebildete Ausdrucksweise" angesiedelt, "die gewisse Kenntnisse und eine gute schulische Ausbildung voraussetzt" und in den genannten Wörterbüchern mit „bildungssprachlich” gekennzeichnet wird. "Es handelt sich hierbei meist um Fremdwörter, die weder einer Fachsprache noch der Umgangssprache angehören" (ebd.). Im Brockhaus-Wahrig wird „bildungssprachlich” den "Angaben zu Sondersprachen" zugeordnet.

Abgesehen davon, daß sich die Zahl der angenommenen "Stilschichten" / „Stilebenen” von Wörterbuch zu Wörterbuch unterscheidet und die Zuordnung von Markierungen $z u$ diesen unterschiedlich ist, schwankt die Zuordnung von diastratischen Markierungen zu Wörtern bzw. zu einzelnen Verwendungsweisen lexikalischer Einheiten erheblich (vgl. Braun 1981, S. 169ff.; Wiegand/Kučera 1981, S. 141ff.; Hausmann 1989, S. 649ff; Corbin 1989, S. 673ff.). Braun (1981, S. 172) hat beispielsweise bei einem Vergleich der Zuordnung von Wörtern zu Stilschichten im WDG und Duden-GWB (1976) festgestellt, daß „die untere Grenze der Abweichungen [...] bei 30\%, die obere bei 70\%" liegt. Eine zur Zeit laufende, aber noch nicht abgeschlossene Untersuchung der Zuordnung von diastratischen und diaevaluativen Markierungen zu Wörtern im WDG und HDG läßt eine ähnliche Tendenz in der unterschiedlichen stilistischen Markierung erkennen. Nach vorläufiger Auswertung ist innerhalb der Buchstabenstrecke $A$ bis $K$, in der rund 8400 Wörter oder deren Verwendungsweisen eine "stilistische" Markierung aufweisen, eine Differenz der Zuordnung zu Stilschichten von etwa $40 \%$ festzustellen. Das liegt zum Teil daran, daß die von den im WDG als „dichterisch" gekennzeichneten lexikalischen Einheiten, die auch in das HDG übernommen worden sind, hier meist als "gehoben” markiert werden, häufig auch als "veraltend gehoben” (vgl. hierzu Tabelle 3). Als „dichterische Wörter und Redewendungen" werden im WDG lexikalische Einheiten gekennzeichnet, „die im allgemeinen der poetischen Gestaltung eines Werkes vorbehalten sind" (WDG, S. 012). Im HDG werden zur "gehobenen Stilebene" Lexeme gerechnet, „die gewählt, feierlich, erhaben anmuten” (HDG, S. XXII). Im Duden-UW, das bei diesem Vergleich mit herangezogen worden ist, werden diese Lexeme häufig - wie im WDG - als „dichterisch” bewertet. Es sind „Wörter, die vorzugsweise in der älteren Dichtung vorkommen 
und heute fast durchgängig veraltet sind oder altertümelnd gebraucht werden" (Duden-UW 1989, S. 9).

Beim Vergleich der Zuordnung lexikalischer Einheiten zu Stilschichten ist vom WDG zum HDG hin sehr häufig die Tendenz einer "Aufwertung” festzustellen. Das heißt: Wörter bzw. bestimmte Verwendungsweisen lexikalischer Einheiten, die im WDG als "salopp" gekennzeichnet sind, erhalten im HDG das Markierungsprädikat „umgangssprachlich”; im WDG als „umgangssprachlich" markierte Lexeme erscheinen im HDG mit „Nullmarkierung”. Wir haben auch bei diesem Vergleich die jeweils im Duden-UW verzeichneten Markierungen mit herangezogen. 


\begin{tabular}{|c|c|c|c|}
\hline & \multicolumn{3}{|c|}{$\begin{array}{l}\text { Stilistische Markierung } \\
\text { in den Wörterbüchern }\end{array}$} \\
\hline Lemmabeispiele & WDG & HDG & Duden-UW \\
\hline Aar (Adler) & dicht. & veraltend geh. & dichter. veraltet \\
\hline Altvorder(e)n (Vorfahren) & dicht. & noch scherzh. & veraltet, geh. \\
\hline Barke (kleines Boot ohne Mast) & dicht. & - & - \\
\hline beglänzen (Glanz auf etw. werfen) & dicht. & - & dichter. \\
\hline benetzen (anfeuchten) & dicht. & geh. & geh. \\
\hline $\begin{array}{l}\text { Brandmal } \\
\text { (das unauslöschliche } \\
\text { Zeichen) }\end{array}$ & dicht. & geh. & \begin{tabular}{|l} 
nicht in dieser \\
Bedeutung \\
verzeichnet \\
\end{tabular} \\
\hline Busen (Brust des Menschen) & dicht. & veraltend & dichter., veraltet \\
\hline Dämmerschein & dicht. & geh. & geh. \\
\hline dräuen (drohen) & dicht. & veraltend geh. & dichter. \\
\hline Eiland (Insel) & dicht. & veraltend geh. & dichter. \\
\hline $\begin{array}{l}\text { fächeln } \\
\text { (in sanfter Bewegung wehen) }\end{array}$ & dicht. & geh. & geh. \\
\hline Fittich (Flügel) & dicht. & geh. & dichter. \\
\hline Gefilde (Landschaft) & dicht. & geh. & geh. \\
\hline gülden (golden) & dicht. & geh. & $\begin{array}{l}\text { dichter., } \\
\text { sonst meist iron. }\end{array}$ \\
\hline Hain (kleiner Wald) & dicht. & geh. & dichter. veraltet \\
\hline hehr (erhaben) & dicht. & veraltend & geh. \\
\hline Himmelszelt (Himmel) & dicht. & geh. & dichter. \\
\hline Hort (Schatz) & dicht. & veraltend & dichter. \\
\hline $\begin{array}{l}\text { Jungbrunnen (etw., was jmdm. } \\
\text { neuen Schwung verleiht) }\end{array}$ & dicht. & - & - \\
\hline künden (berichten) & dicht. & geh. & geh. \\
\hline Lenz (Frühling) & dicht. & geh. & dichter. \\
\hline Nachen (Kahn, Boot) & dicht. & veraltend geh. & dichter. \\
\hline Odem (Atem) & dicht. & veraltend geh. & dichter. \\
\hline Parnaß (Reich der Dichtkunst) & dicht. & veraltend geh. & dichter. geh. \\
\hline Port (Ort der Geborgenheit) & dicht. & veraltend geh. & dichter. veraltet \\
\hline
\end{tabular}

Tabelle 3: Beispiele für die Zuordnung von Lexemen zu den Markierungsprädikaten "dichterisch" und "gehoben" in drei deutschen Wörterbüchern

$\begin{array}{ll}- & =\text { ohne Markierungsprädikat ( Nullmarkierung") } \\ \text { dicht./dichter. } & =\text { dichterisch } \\ \text { geh. } & =\text { gehoben } \\ \text { iron. } & =\text { ironisch }\end{array}$


Wie bereits erwähnt, wird im WDG eine „Variante der Normalsprache, die im mündlichen Gebrauch erscheint und für die einige Wörter (z.B. kriegen 'bekommen') typisch sind", als "Umgangssprache" bezeichnet. „Schriftlich werden diese Wörter nur in privaten Briefen gebraucht sowie in der Literatur, um eine gewisse Vertraulichkeit auszudrücken”, sie erhalten die "Bewertung umgangssprachlich" (WDG, S. 012). Im HDG werden zur "umgangssprachlichen Stilebene" Lexeme gerechnet, "die in einer zwanglosen Sprache des alltäglichen mündlichen oder (nichtöffentlichen) schriftlichen Gebrauchs verwendet werden" (HDG, S. XXII). Im Duden-UW (1989, S. 9) wird darauf verwiesen, daB der „normalsprachlichen Stilschicht” die "Umgangssprache” nahesteht, „die im alltäglichen, besonders im familiär-vertraulichen, mündlichen Verkehr der Menschen untereinander üblich ist und in Briefen verwendet wird. Sie ist aber heute auch häufig in der Öffentlichkeit anzutreffen und hat Eingang in die Literatur gefunden." Die „saloppumgangssprachliche" Schicht unterscheidet sich nach dem WDG „von der Normalsprache, einschließlich ihrer umgangssprachlichen Variante, durch eine gewisse Nachlässigkeit” und ist ,im*alltäglichen Verkehr der Menschen untereinander heute sehr verbreitet. [...] Sie würde, im öffentlichen Leben verwendet, salopp und leicht anstöBig wirken. [...] Die Wörter und Redewendungen dieser Stilschicht sind mehr oder weniger gefühlsbetont” (WDG, S. 012). Im HDG werden der „saloppen Stilebene” Lexeme zugeordnet, „die eine legere Haltung des Sprechers ausdrücken, oft bildhaft sind und eine starke emotionale Aufladung besitzen" (HDG, S. XXII). Als „salopp” kennzeichnet das Duden-UW $(1989$, S. 9) Wörter und Verwendungsweisen, "wie sie für eine burschikose und z.T. recht nachlässige Ausdrucksweise charakteristisch sind."

Im WDG als „salopp”, in HDG und Duden-UW als „umgangssprachlich” werden z. B. gekennzeichnet:

abdampfen (weggehen, -fahren), abkanzeln (scharf tadeln), Abreibung (scharfe Zurechtweisung; Prügel), sich abstrampeln (sich abmühen), etw. ist amtlich (ganz sicher), angesäuselt (ein wenig betrunken), Bärenhunger (großer Hunger), Bauchkneipen (Bauchschmerz), Bauchladen, Hals- und Beinbruch (alles Gute!), beschummeln (ein wenig betrügen), bibbern (zittern), blümerant (unwohl), bockbeinig (störrisch), einen Bock schießen (einen Fehler machen), dazwischenfunken (dazwischenfahren), dickfellig (gleichgültig), der, die, das Dings (Person; irgend etwas; Ortsname), dösen (mit wachen Augen vor sich hin träumen; leicht schlafen), durchackern (etw. durcharbeiten), durchbrennen (sich heimlich davonmachen), etw. aus dem Effeff (vorzüglich) können, beherrschen; einfädeln (anbahnen), Flittchen, Ganove, Gaudi (Spaß), junges Gemüse (unerfahrene Jugendliche), Job (Beschäftigung), Käfer (hübsches junges Mädchen), knutschen. 
Im WDG als „umgangssprachlich” gekennzeichnete lexikalische Einheiten erscheinen in HDG und Duden-UW in vielen Fällen mit „Nullmarkierung”, z.B.:

abgekämpft (ermũdet), abgemacht! (es gilt), sich abhetzen (sich sehr beeilen), abreagieren (Affekte entladen), adrett (sauber und ordentlich), ahnungslos (völlig unwissend), Akku (Akkumulator), Amüsement (Vergnügen), das ist Ansichtssache (darüber kann man verschiedener Meinung sein), bemuttern (umsorgen), Binsenwahrheit (allgemein bekannte Tatsache), blendend (ausgezeichnet), brühheiß (sehr heiß), brutto (ohne Abzug der Abgaben, Steuern), Bus (Omnibus), Busfahrer, Bushaltestelle, couragiert (beherzt), deplaciert (unangebracht), Desaster (schweres MiBgeschick, Zusammenbruch), dummerweise (ärgerlicherweise; törichterweise), Dummkopf (dummer Mensch), das Eingemachte (eingemachte Lebensmittel), en passant (beiläufig), Faible (Vorliebe), feuchtfröhlich (fröhlich, ausgelassen durch AlkoholgenuB), Flickzeug (Material zum Flicken), fortbringen (wegbringen), fortschaffen (wegbringen), Galgenfrist (Gnadenfrist), Geläster (dauerndes Lāstern), Glückskind (jmd., der immer Glück hat), hänseln (sich über jmdn. lustig machen), herumtollen (ausgelassen umherspringen, -laufen), humpeln (mühsam gehen), Ischias (Schmerzen im Bereich des Ischiasnervs), Jammergestalt (mitleiderregender Mensch), johlen (lärmend schreien), Kalamität (Schwierigkeit), Kalauer (wenig geistreiches Wortspiel), Kleinigkeitskrämer (jmd., der Kleinigkeiten übertrieben wichtig nimmt).

Bemerkenswert scheint mir bei dem Vergleich der Zuordnung von sprachlichen Ausdrücken zu Stilschichten im WDG bzw. Stilebenen im HDG die Tatsache, daB ein Dutzend Mitarbeiter des Autorenkollektivs des HDG auch Autoren des WDG gewesen sind. Haben sich also die Bewertungsnormen vom AbschluB des WDG über die Erarbeitungsphase des HDG verändert?

Weitgehend übereinstimmen die im WDG als "vulgär" markierten Wörter mit denen, die im HDG der Stilebene "derb" zugeordnet sind. Nach dem WDG gehören zur Schicht vulgärer Wörter und Redewendungen solche, "die als ausgesprochen grob empfunden und deshalb im allgemeinen vermieden werden”. Sie sind „nur in beschränktem Umfang in dieses Wörterbuch aufgenommen." (WDG, S. 012). Es wird ausdrücklich darauf aufmerksam gemacht, daß obszöne Wörter, die zur vulgären Schicht gehören, nicht berücksichtigt sind. Im HDG werden der „derben Stilebene" Lexeme zugeordnet, die ,in hohem Grade drastisch, verletzend oder unter Umständen anstößig wirken" (HDG, S. XXII).

\subsection{Zur Markierungspraxis im Duden (Rechtschreibwörterbuch)}

In der in jüngster Zeit geführten Diskussion über die Mikrostruktur der Wörterbuchartikel eines Rechtschreibwörterbuches geht es auch um die Frage, welche Angaben zu einem Lemma bzw. Lemmazeichen aufzunehmen sind. „Grundsätzlich gilt für die Prüfung [...] das Krite- 
rium der rechtschreiblichen Relevanz; d.h., es sollen nur solche Angaben Eingang in einen Wörterbuchartikel finden, die in irgendeiner Weise die Rechtschreibung des jeweiligen Wortes betreffen. Bisher ist nicht endgültig ausgemacht, welche Arten von Angaben dies im Einzelfall sind" (Augst/Schaeder 1991, S. 90). Reichlich Gebrauch gemacht wird heute im Duden von den diasystematischen Markierungen, die nach Herberg (1993, S. 348) im Rahmen des Konzepts von Zentrum und Peripherie zur Peripherie der Informationen eines im Rechtschreibwörterbuch verzeichneten Lemmas zu zählen sind. Ich möchte nicht darüber sinnieren, ob der Duden vom „Universalwörterbuch”, "Mehrzweckwörterbuch”, "Allzweckwörterbuch”, „Überwörterbuch” (Nerius 1988, S. 478f.; 1988a, S. 252f.) wieder zum reinen Rechtschreibwörterbuch zurückkehren sollte. Aber: Sollte der Duden auch zukünftig die vielfältigen nichtorthographischen Kennzeichnungen zu Lemmata beibehalten, so sollten - nein - müßten diese Angaben zu einer präziseren, besser durchschaubaren Praxis führen (vgl. auch Nerius 1988a, S. 253f.). Und das betrifft - und hierauf beschränke ich mich - auch die „stilistische" Kennzeichnung von Wörtern im Duden, die Zuordnung zu "Stilschichten” und/oder "Stilfärbungen” (nach der Terminologie allgemeiner einsprachiger Wörterbücher).

Weder in der letzten Ausgabe des Leipziger Dudens (18. Neubearbeitung 1985) noch in der letzten Ausgabe des Mannheimer Dudens (19. Auflage 1986), noch in dem 1991 erschienenen "wiedervereinigten" Wörterbuch zur deutschen Rechtschreibung (20. Auflage Mannheim/Leipzig) werden stilistische Markierungen in den jeweiligen Hinweisen für den Benutzer thematisiert, wenn man von folgenden allgemeinen Bemerkungen absieht:

Im "Vorwort” zur 18. Neubearbeitung des Leipziger Dudens (1985, S. 5) heiBt es: „Die stilistischen Angaben wurden im gesamten Wörterverzeichnis nach einheitlichen Kriterien bearbeitet." Zum „Aufbau der Stichwortartikel" der "Einführung in Anlage und Aufbau des Wörterverzeichnisses" ist zu lesen: "Die Worterläuterungen stehen in sunden Klammern, wenn nötig, mit kursiven Sachgebiets-, territorialen (landschaftlichen) oder stilistischen Kennzeichnungen versehen. [...] Die stilistischen Angaben wurden häufig relativiert, da eine absolute Kenzzeichnung ohne Kontext oft nicht möglich ist" (S. 9f.). Bleiben die Tragen: Was sind eigentlich stilistische Angaben/Kennzeichnungen? Nach welchen einheitlichen Kriterien wurden sie bearbeitet?

Im „Vorwort” und in den „Hinweise[n] für den Benutzer” des Mannheimer Dudens von 1986 finden sich keine Ausführungen zu den stilistischen Markierungen. Im "Vorwort” der Neuausgabe des Dudens von 1991 wird 
(wohl) zumindest auf derartige Angaben hingewiesen, wenn es heißt: „Da der Duden nicht nur ein Rechtschreibbuch ist, sondern auch die Funktion eines Volkswörterbuchs erfüllt, wurden die Erklärungen schwieriger Wörter, vor allem veraltender, landschaftlicher, umgangssprachlicher und fachsprachlicher Ausdrücke, und die Angaben zum Wortgebrauch beträchtlich erweitert” (S. 6). Keine Erläuterungen dazu, was „Angaben zum Wortgebrauch" sind, inwiefern diese Angaben beträchtlich erweitert wurden und welches Bewertungssystem diesen Angaben, mit denen vermutlich auch "stilistische Kennzeichnungen” gemeint sind, zugrunde liegt.

Nur über das jeweilige Abkürzungsverzeichnis und die im Wörterverzeichnis verwendeten Markierungsprädikate ist $\mathrm{zu}$ erschließen, welche Angaben eigentlich "stilistische Angaben” sind; sie werden nicht systematisch eingeführt. Das betrifft nicht nur Markierungen, die in allgemeinen einsprachigen Wörterbüchern zu "Stilschichten”/ „Stilebenen” gehören (s. Tabelle 4), sondern auch Angaben, die "Stilfärbungen” bzw. "Gebrauchsangaben" zugeordnet werden (z.B. "scherzhaft”, ,spöttisch", "ironisch", „abwertend").

\begin{tabular}{|c|c|c|}
\hline \multicolumn{2}{|c|}{$\begin{array}{c}\text { Markierungen im Duden, die "Stilschichten"/,Stilebenen" } \\
\text { in allgemeinen einsprachigen Wörterbüchern } \\
\text { des Deutschen entsprechen }\end{array}$} \\
\hline $\begin{array}{c}\text { Duden 1985 } \\
\text { (18. Neubearbeitung } \\
\text { Leipzig) }\end{array}$ & $\begin{array}{c}\text { Duden 1986 } \\
\text { (19. Aufl. Mannheim) }\end{array}$ & $\begin{array}{c}\text { Duden 1991 } \\
\text { (20. Aufl. } \\
\text { Mannheim/Leipzig) }\end{array}$ \\
\hline $\begin{array}{c}\text { dichterisch } \\
\text { gehoben } \\
\text { normalsprachlich } \\
\text { umgangssprachlich } \\
\text { salopp } \\
\text { derb }\end{array}$ & $\begin{array}{c}\text { dichterisch } \\
\text { gehoben }\end{array}$ & gehoben \\
\hline
\end{tabular}

Tabelle 4: Markierungen für Stilschichten/Stilebenen in drei Dudenausgaben

Kurios ist, daß im Abkürzungsverzeichnis des Leipziger Dudens (1985) die Angabe „normalsprachlich” auftaucht. Bisher habe ich im Wörterverzeichnis dieser Dudenausgabe kein so markiertes Lemma entdeckt. Sowohl in der Leipziger Ausgabe von 1985 als auch in den Dudenausgaben von 1986 und 1991 haben wir jeweils auch die „Nullmarkierung” bei Lemmata, die nicht explizit (d.h. durch ein verbales Prädikat) markiert sind. Ohne hier auf Einzelheiten unterschiedlicher stilistischer Markierungen von lexikalischen Einheiten in den genannten drei Dudenausgaben ein- 
gehen zu können (vgl. Ludwig 1994), sei zu den bisherigen Ergebnissen einer laufenden Untersuchung nur soviel mitgeteilt:

Die sowohl im Leipziger Duden (1985) als auch im Mannheimer Duden (1986) als "dichterisch" markierten Lemmata werden im "Einheitsduden” (1991) als "gehoben" gekennzeichnet, z.B.: Aar (Adler), Allmutter('Mutter alles Lebenden': Allmutter Natur), Fittich (Flügel), Lenz (Frühling), Odem (Atem).

Die Markierung „salopp” wird nur im Leipziger Duden (1985) verwendet. Die hier so gekennzeichneten Lemmata werden im Mannheimer Duden von 1986 und im Duden von 1991 als "umgangssprachlich" markiert, z.B.: aasen (verschwenderisch umgehen), abgetakelt (heruntergekommen, ausgedient), abhauen (davonlaufen), ausgekocht (durchtrieben), auswischen ( $j m d m$. eins auswischen 'schaden').

Nahezu Übereinstimmung herrscht bei den als „derb” markierten Lemmata, 2.B.: Anschiß (Tadel), Arsch, Arschkriecher, auskotzen, ficken, Scheiße.

\section{Ausblick}

Im Vorwort der annotierten Bibliographie zur Theorie der Lexikographie "Lexicography Today" (1988) macht Zgusta im Zusammenhang mit terminologischen Fragen nachdrücklich darauf aufmerksam, daß die größte terminologische und begriffliche Unklarheit im Bereich der diasystematischen Markierungen im Wörterbuch besteht und innerhalb dieses Gebietes vor allem die stilistischen betrifft (S. XII).

An Überlegungen und Vorschlägen zur Verbesserung der bisherigen Praxis stilistischer Markierung in Wörterbuch mangelt es nicht (vgl. z.B. Rossipal 1973, Wiegand 1981, Schumann 1983, Ludwig 1991). Thesenhaft möchte ich die Problematik der "Angaben zum Stil” im Wörterbuch zusammenfassen und hierbei einen eigenen Vorschlag zur Verbesserung der bisherigen Praxis einbeziehen:

(1) Es gibt bestimmte Wörter und Wendungen, die im Vergleich zu anderen a uffällig sind. Erinnern wir uns an die eingangs erwähnten Wörter Visage - Gesicht, denen wir auch die Synonyme Angesicht Antlitz - Fresse hinzufügen können. Oder nehmen wir Überschrift und Inhalt eines Artikels in der „Zeit” (11. März 1994, S. 93): „Wie flucht Europa? Und wie redet es sich heraus? Ein Brite hat die Unterschiede analysiert. Merde, Scheiße, shit” in der Rubrik "Modernes Leben”. Über das deutsche Wort schreibt die Autorin Roggenkamp: „Das Wort hat selbstverständlich in jedem europäischen Land seine Bedeutung, nimmt 
aber nirgendwo diesen Rang ein" (wie bei den Deutschen). Dies paBt zu der Frage einer Studentin, die nach dem Seminar, in dem wir die hier zur Debatte stehende Problematik erörtert hatten, zu mir kam und mich fragte: „MuB denn dieses Wort im Wörterbuch überhaupt gekennzeichnet werden? Jeder gebraucht es, auch die Professoren!" Es gibt also auffällige Wörter. Hausmann (1989, S. 649) hat darauf hingewiesen, daß mit einer solchen Auftälligkeit eine „unmittelbare Auffälligkeit gemeint” ist und nicht eine, „die erst nach eingehender linguistischer Analyse zutage tritt. Solche Auffälligkeit ergibt sich durch ein gegenüber der Normalität zusätzliches Merkmal, das der sprachlichen Einheit wie ein Etikett anhaftet. Wir sagen, die sprachliche Einheit ist markiert. Dem Durchschnittsphänomen fehlt dieses Merkmal, es ist unmarkiert." Und besonders wichtig ist Hausmanns Zusatz: „Für die Textproduktion ergeben sich aus der Markiertheit Verwendungsrestriktionen, Wortverwendungsgrenzen, um die der Muttersprachler intuitiv weiB, die der Fremdsprachler jedoch, ganz so wie die phonetischen, morphologischen, semantischen, syntagmatischen und paradigmatischen Regeln, eigens lernen muB."

DaB diese Auffalligkeit der Wörter im Wörterbuch kenntlich zu machen ist, ist wohl unbestritten. Die Fragen sind: Wie sind diese „Auffalligkeiten” oder „Zusätze” kenntlich zu machen? Wie sind diese „Etiketten” zu beschriften? Damit im Zusammenhang steht das Problem: Wie viele „Etiketten" sollten verwendet werden?

(2) Meines Erachtens geht es in erster Linie darum, dem Wörterbuchbenutzer Informationen darüber zu vermitteln, ob eine lexikalische Einheit im Rahmen einer kommunikativen Prädisposition als „neutral” anzusehen ist, also keinerlei "Auffäligkeit" und somit auch keinerlei Verwendungsrestriktionen aufweist, oder ob die lexikalische Einheit nach "oben" ("über neutral”) oder nach „unten" („unter neutral”) von der neutralen Ebene abweicht und somit Gebrauchsrestriktionen unterliegt. Mit diesen Informationen in Form von "Markierungsetiketten" soll das im Alltagsverständnis der Sprecher vorhandene Wissen über Verwendungsmaximen von Lexemen lexikographisch erfaßt werden. Was ist mit k o m m u n k t iver Prädis position gemeint? Da die Verwendung der Termini „Stilschicht”/ „Stilebene” und "Stilfärbung" als Stilbewertungskategorien insofern problematisch ist, als "Stil" gewöhnlich als textimmanente Eigenschaft begriffen wird, „Stilschicht"/nStilebene" und "Stilfärbung" jedoch auf ein Einzellexem bzw. eine seiner spezifischen Verwendungsweisen bezogen werden, habe ich vorgeschlagen, anstelle dieser Termini den Begriff der kommunikativen Prädisposition zu verwenden (vgl. Ludwig 1991, S. 222ff.). Damit soll ausgedrückt werden, daß eine lexikalische Ein- 
heit prädisponiert ist, in bestimmten Bereichen der Kommunikation gebraucht zu werden. Lexikalische Einheiten, die dieselbe kommunikative Prädisposition aufweisen, d.h. ohne Einschränkung bzw. nur mit Einschränkung verwendet werden können, gehören dann zur selben $k$ ommunikat iven Prädispositionsebene; sie gibt den allgemeinen Bereich der Kommunikation an, in dem die entsprechende lexikalische Einheit vorwiegend gebraucht werden kann. Die Ebenen dieses dreistufigen Modells stellen zunächst ein grobes, aber grundlegendes Raster für die Einordnung lexikalischer Einheiten in Verwendungsbereiche und die entsprechenden Markierungen damit Hinweise für Verwendungsrestriktionen dar, wobei zu erwägen ist, die Ebene „unter neutral” zu differenzieren in "umgangssprachlich” bzw. „ungezwungen” und "derb”. Hinzu kommen können weitere Markierungen, die spezifische Hinweise für Gebrauchsmöglichkeiten eines Lexems geben, "Gebrauchsangaben”, die „etwas über die Haltung des Sprechers oder die Nuancierung einer Äußerung aussagen" (Duden-GWB 1993, S. 20). So können z.B. Markierungen darauf hinweisen, daß durch die Verwendung dieses Lexems der Sprecherstandpunkt, insbesondere eine emotionale Einstellung des damit bezeichneten Denotats ausgedrückt werden kann. Andere Markierungen weisen darauf hin, daß durch den Gebrauch lexikalischer Einheiten in bestimmten Zusammenhängen insofern die Sprecherintention angedeutet wird, als damit eine gewisse Scherzhaftigkeit oder Distanzierung zum Ausdruck gebracht werden kann.

(3) Um eine ausgewogene und systematische „stilistische” Markierung der Einzellexeme in einem Wörterbuch zu erzielen, ist es unabdingbar, das Einzellexem jeweils mit anderen Lexemen des entsprechenden Lexemfeldes in Beziehung zu setzen, d.h., eine solche ausgewogene und systematische „stilistische” Bewertung kann nur im Feldzusammenhang geschehen, was einen Vergleich kompletter Synonymfelder voraussetzt. Zu prüfen ist ebenfalls, ob zu dem als „über neutral” bzw. „unter neutral" zu markierenden Lexem jeweils ein Synonym vorhanden ist, das der „neutralen” Ebene zugeordnet werden kann.

(4) Man sollte versuchen, die im Wörterverzeichnis verwendeten Markierungen auf eine begründbare Typologie zurückzuführen, damit dem Lexikographen ein $\mathrm{m} \ddot{\mathrm{g}} \mathrm{li} \mathrm{ch} \mathrm{s} \mathrm{t}$ einheitliches Beschreibungsinventar zur Verfügung steht. Daß sich mit „einem geschlossenen Inventar von Prädikatsausdrücken [...] die vielfältigen Nuancen im Wortgebrauch nicht erfassen" lassen (Püschel 1989, S. 698), ist ebenso unbestritten wie auch die Beschreibung dieser Nuancen in Form sogenannter "offener Kommentare”, die Wiegand (1981) vorgeschlagen hat, nicht ausgeschlossen wer- 
den soll. Die wiederholt erhobene Forderung, im Wörterbuch genaue Regelbeschreibungen für den situationsspezifischen Gebrauch von Lexemen anzugeben, besteht sicherlich zu Recht. Sie ist jedoch insofern schwer zu erfüllen, als hierbei von konkreten Situationen zu abstrahieren ist, um zu Typen von Situationen zu gelangen. Es bleibt weiterhin zu bedenken: Je mehr Markierungsprädikate für die stilistische Bewertung verwendet werden, um so größer werden gleichzeitig die Schwierigkeiten der Lexikographen bei der Kennzeichnung von Lexemen. Wichtig ist allerdings: Dem Benutzer muB der Bewertungsmodus von lexikalischen Einheiten in den Benutzungshinweisen erklärt werden, und es müssen sämtliche im Wörterverzeichnis verwendeten Markierungen in der Wörterbucheinleitung eingeführt und umfassend beschrieben werden.

\section{Schlußbemerkung}

Eine endgültige, alle Wörterbuchbenutzer und Linguisten befriedigende Lösung in bezug auf die ,stilistische" Bewertung und Kennzeichnung von lexikalischen Einheiten im Wörterbuch wird es nicht geben. Den Markierungen ist „kein absoluter, wohl aber ein relativer Wert zuzumessen" (Hausmann 1989, S. 650), denn „das Sprachgefühl ist bei den Menschen nicht immer das gleiche, es kann divergieren je nach Alter, Bildung oder Landschaft, aus der ein Sprecher kommt" (Klappenbach 1964/1980, S. 93).

\section{Literatur}

1. Wörterbücher:

Adelung, Johann Christoph (1774-1786): Versuch eines vollständigen grammatisch-kritischen Wörterbuches Der Hochdeutschen Mundart, mit beständiger Vergleichung der übrigen Mundarten, besonders aber der Oberdeutschen. 5 Bde. Leipzig.

Brockhaus-Wahrig (1980-1984): Deutsches Wörterbuch in sechs Bänden. Hrsg. von Gerhard Wahrigt, Hildegard Krämer und Harald Zimmermann. Wiesbaden/Stuttgart.

Bußmann, Hadumod (1990): Lexikon der Sprachwissenschaft. 2., völlig neu bearb. Aufl. Stuttgart.

Campe, Joachim Heinrich (1807-1811): Wörterbuch der Deutschen Sprache. 5 Bde. Braunschweig.

Conrad, Rudi (Hrsg.) (1985): Lexikon sprachwissenschaftlicher Termini. Leipzig.

Duden. Bedeutungswörterbuch (1985): Duden. Bedeutungswörterbuch. 2., völlig neu bearb. und erw. Aufl. Hrsg. und bearb. von Wolfgang Müller. Mannheiun/Wien/Zürich. 
Duden (1985): Der Große Duden. Wörterbuch und Leitfaden der deutschen Rechtschreibung. 18. Neubearbeitung. Leipzig.

Duden (1986): Duden. Rechtschreibung der deutschen Sprache und der Fremdwörter. 19., neu bearb. und erw. Aufl. Mannheim/Wien/Zürich.

Duden (1991): Duden. Rechtschreibung der deutschen Sprache. 20., völlig neu bearb. und erw. Aufl. Mannheim/Leipzig/Wien/Zürich.

Duden-GWB (1976-1981): Duden. Das große Wörterbuch der deutschen Sprache in sechs Bänden. Hrsg. und bearb. vom Wissenschaftlichen Rat und den Mitarbeitern der Dudenredaktion unter Leitung von Günther Drosdowski. Mannheim/Wien/Zürich.

Duden-GWB (1993): Duden. Das große Wörterbuch der deutschen Sprache. In acht Bänden. 2., völlig neu bearb. und stark erw. Aufl. Hrsg. und bearb. vom Wissenschaftlichen Rat und den Mitarbeitern der Dudenredaktion unter Leitung von Günther Drosdowski. Bd. 1-3. Mannheim/Leipzig/Wien/Zürich.

Duden-UW (1989): Duden. Deutsches Universalwörterbuch. 2., völlig neu bearb. und stark erw. Aufl. Hrsg. und bearb. vom Wissensch aftlichen Rat und den Mitarbeitern der Dudenredaktion unter Leitung von Günther Drosdowski. Mannheim/Wien/Zürich (1. Aufl. 1983).

HDG (1984): Handwörterbuch der deutschen Gegenwartssprache. In zwei Bānden. Von einem Autorenkollektiv unter der Leitung von Gūnter Kempcke. Berlin.

Knaur (1985): Knaur. Das deutsche Wörterbuch. Erarbeitet von Ursula Hermann unter Mitarbeit von Horst Leisering und Heinz Hellerer. München.

Krahl, Siegfried/Kurz, Josef (1984): Kleines Wörterbuch der Stilkunde. 6., neubearb. Auft. Leipzig.

Lewandowski, Theodor (1990): Linguistisches Wörterbuch. 5., überarb. Aufl. Heidelberg/Wiesbaden.

Metzler-Lexikon Sprache (1993): Metzler-Lexikon Sprache. Hrsg. von Helmut Glück. Stuttgart/Weimar.

Sachwörterbuch für die deutsche Sprache (1989): Sachwörterbuch für die deutsche Sprache. Von einem Autorenkollektiv unter Leitung von Karl-Ernst Sommerfeldt und Wolfgang Spiewok. Leipzig.

Wahrig-DW (1986): Deutsches Wörterbuch. Mit einem „Lexikon der deutschen Sprachlehre". Hrsg. in Zusammenarbeit mit zahlreichen Wissenschaftlern und anderen Fachleuten. Völlig überarb. Neuausgabe. München.

WDG (1961-1977): Wörterbuch der deutschen Gegenwartssprache. Hrsg. von Ruth Klappenbach und Wolfgang Steinitz. 6 Bde. Berlin. 


\section{Sekundärliteratur:}

Augst, Gerhard/Schaeder, Burkhard (1991): Rechtschreibwörterbücher der deutschen Gegenwartssprache - Analyse, Kritik, Vorschläge. In: Augst, Gerhard/Schaeder, Burkhard (Hrsg.): Rechtschreibwörterbücher in der Diskussion. Geschichte - Analyse - Perspektiven. Frankfurt a.M./Bern/ New York/Paris. S. 31-98. (= Theorie und Vermittlung der Sprache, Bd. 13).

Braun, Peter (1981): Zur Praxis der Stilkennzeichnungen in deutsch-deutschen Wörterbüchern. In: Muttersprache 91, H. 3-4, S. 169-177.

Corbin, Pierre (1989): Les marques stylistique/diastratiques dans le dictionnaire monolingue. In: Wörterbücher (1989), S. 673-680.

Hausmann, Franz Josef (1977): Einführung in die Benutzung der neufranzösischen Wörterbücher. Tübingen. (= Romanistische Arbeitshefte; 19).

Hausmann, Franz Josef (1989): Die Markierung im allgemeinen einsprachigen Wörterbuch: eine Übersicht. In: Wörterbücher (1989), S. 649-657.

Henne, Helmut (Hrsg.) (1975): Deutsche Wörterbücher des 17, und 18. Jahrhunderts. Einführung und Bibliographie. Hildesheim/New York.

Herberg, Dieter (1993): Deutsche Rechtschreibwörterbücher - wie sie sind und wie sie sein sollten. Aus AnlaB des Erscheinens der 20. Duden-Auflage. In: Zeitschrift für germanistische Linguistik 21.3, S. 334-353.

Klappenbach, Ruth (1964/1980): Das Wörterbuch der deutschen Gegenwartssprache. In: Studien zur modernen deutschen Lexikographie. Hrsg. von Werner Abraham unter Mitwirkung von Jan F. Brand. Amsterdam 1980. S. 89-107.

Klappenbach, Ruth†/Malige-Klappenbach, Helene (1978): Das Wörterbuch der deutschen Gegenwartssprache. Entstehung, Werdegang, Vollendung. In: Kopenhagener Beiträge zur Germanistischen Linguistik 14. S. 5-46.

Ludwig, Klaus-Dieter (1991): Markierungen im allgemeinen einsprachigen Wörterbuch des Deutschen. Ein Beitrag zur Metalexikographie. Tübingen (= Lexicographica. Series Maior, Bd. 38).

Ludwig, Klaus-Dieter (1994): Probleme der Markierung im Wörterbuch. In: Symposium on Lexicography VI. Proceedings of the Sixth International Symposium on Lexicography May 7 - 9, 1992 at the University of Copenhagen. Ed. by Karl Hyldgaard-Jensen and Viggo Hjørnager Pedersen. Tübingen. S. 51-71. (= Lexicographica. Series Maior, Bd. 57).

Müller, Wolfgang (1984): Zur Praxis der Bedeutungserklärung (BE) in (einsprachigen) deutschen Wörterbüchern und die semantische Umkehrprobe. In: Wiegand, Herbert Ernst (Hrsg.): Studien zur neuhochdeutschen Lexikographie V. Hildesheim/Zürich/New York. S. 359-461. (= Germanistische Linguistik 3 - 6/84).

Nerius, Dieter (1988): Aufgaben und Möglichkeiten orthographischer Wörterbücher. In: Symposium on Lexicography III. Proceedings on the Third International Symposium on Lexicography May $14-16,1986$ at the University of Copenhagen. Ed. by Karl Hyldgaard-Jensen und Arne Zettersten. Tübingen. S. 469-484. (= Lexicographica. Series Maior, Bd. 19). 
Nerius, Dieter (1988a): Zur Geschichte und Funktion des Dudens. In: Symposium on Lexicography IV. Proceedings of the Fourth International Symposium on Lexicography April $20-22,1988$ at the University of Copenhagen. Ed. by Karl Hyldgaard-Jensen und Arne Zettersten. Tübingen. S. 249-264. (= Lexicographica. Series Maior, Bd. 26).

Pūschel, Ulrich (1989): Evaluative Markierungen im allgemeinen einsprachigen Wörterbuch. In: Wörterbücher (1989), S. 693-699.

Roggenkamp, Viola (1994): Wie flucht Europa? Und wie redet es sich heraus? Ein Brite hat die Unterschiede analysiert. Merde, Scheiße, shit. In: Die Zeit, Jg. 49, Nr. 11 (11. März 1994), S. 93.

Rossipal, Hans (1973): Konnotationsbereiche, Stiloppositionen und die sogenannten "Sprachen" in der Sprache. Hildesheim. (= Germanistische Linguistik 4/73).

Schumann, Hanna Brigitte (1983): Versuch, Konnotativität als kommunikative Präferenz lexikalischer Einheiten mit Hilfe einer Merkmalmatrix zu beschreiben. In: Die Lexikographie von heute und das Wörterbuch von morgen. Analysen - Probleme - Vorschläge. Hrsg. von Joachim Schildt und Dieter Viehweger. Berlin. S. 300-314. (= LS/ZISW/A, H. 109).

Wiegand, Herbert Ernst (1981): Pragmatische Informationen in neuhochdeutschen Wörterbüchern. Ein Beitrag zur praktischen Lexikologie. In: Wiegand, Herbert Ernst (Hrsg.): Studien zur neuhochdeutschen Lexikographie I. Hildesheim/New York. S. 139-271. (= Germanistische Linguistik 3 $-4 / 79)$.

Wiegand, Herbert Ernst/Kučera, Antonín (1981): Brockhaus-Wahrig: Deutsches Wörterbuch auf dem Prüfstand der praktischen Lexikologie. I. Teil: 1. Band (A - BT); 2. Band (BU - FZ). In: Kopenhagener Beiträge zur germanistischen Linguistik 18, S. 94-214.

Wörterbücher (1989): Wörterbücher. Ein internationales Handbuch zur Lexikographie. Hrsg. von Franz Josef Hausmann/Oskar Reichmann/Herbert Ernst Wiegand/Ladislav Zgusta. 1. Teilband Berlin/New York.

Zgusta, Ladislav (1988): Zgusta, Ladislav with the assistance of Donna M.T.Cr. Farina: Lexicography Today. An annotated bibliography of the theory of lexicography. Tübingen. (= Lexicographica. Series Maior, Bd. 18). 